\title{
TECHNICAL SPOTLIGHT \\ Pavlovian fear conditioning as a behavioral assay for hippocampus and amygdala function: cautions and caveats
}

\author{
Stephen Maren \\ Department of Psychology and Neuroscience Program, University of Michigan, 530 Church St., Ann Arbor, MI 48109-1043, USA
}

Keywords: behavioral assay, context, cue, fear responses, learning

\begin{abstract}
Pavlovian fear conditioning has become an important model for investigating the neural substrates of learning and memory in rats, mice and humans. The hippocampus and amygdala are widely believed to be essential for fear conditioning to contexts and discrete cues, respectively. Indeed, this parsing of function within the fear circuit has been used to leverage fear conditioning as a behavioral assay of hippocampal and amygdala function, particularly in transgenic mouse models. Recent work, however, blurs the anatomical segregation of cue and context conditioning and challenges the necessity for the hippocampus and amygdala in fear learning. Moreover, nonassociative factors may influence the performance of fear responses under a variety of conditions. Caution must therefore be exercised when using fear conditioning as a behavioral assay for hippocampal- and amygdala-dependent learning.
\end{abstract}

When Estes and Skinner described the conditioning of 'anxiety' in rats in 1941 (Estes \& Skinner, 1941), they did not foresee that rodent models of aversive conditioning would become one of the most ubiquitous behavioral paradigms to explore the neural substrates of learning and memory. Indeed, Pavlovian fear conditioning has become part of the standard arsenal of behavioral tasks used to interrogate the mnemonic capacities of rats, mice and humans. In fear conditioning, neutral stimuli (conditional stimuli or CSs) such as tones, lights, or places (contexts) are arranged to predict aversive outcomes such as footshock (an unconditional stimulus or US). After conditioning, CSs come to evoke learned fear responses (conditional responses or CRs) such as conditioned suppression, freezing and tachycardia. The explosion of scientific papers on this topic indexes the popularity of fear conditioning. A search of the Institute for Scientific Information Web of Science database reveals an enormous and exponential growth in publications on fear conditioning from a total of 17 papers in 1977 , 12 papers in 1987, 138 papers in 1997 and 381 papers in 2007. The search terms were 'fear conditioning', 'conditioned fear', 'conditioned suppression', 'conditioned freezing', or 'fear-potentiated startle'. The search revealed a total of 4207 hits over the history of the database (1954-2008).

Why has fear conditioning become such a popular paradigm for learning and memory research? First, the equipment required for fear conditioning can be readily scaled to condition several animals simultaneously. Fear conditioning requires nothing more than standard rodent conditioning chambers with grid floors, shock sources and stimulus generators, and some form of recording equipment (e.g.

Correspondence: Dr Stephen Maren, as above.

E-mail: maren@umich.edu

Received 31 July 2008, revised 4 September 2008, accepted 8 September 2008 video cameras, force transducers or photocells) to measure fear responses such as freezing behavior. Estes \& Skinner (1941) indexed fear conditioning by quantifying the degree to which rats would suppress ongoing appetitive behavior (e.g. lever pressing for food), but it is now routine to measure fear behaviors themselves. Several chambers can be operated in unison (e.g. 8-16 chambers in a setup is not uncommon) and a typical multichamber setup requires minimum floor space. Second, fear-conditioning procedures produce rapid, robust and enduring learning; a single, intense footshock produces dramatic fear conditioning that is retained for months. As a consequence, it requires only a few minutes to condition fear in several rats simultaneously; testing the retention of that memory is similarly rapid. Third, humans readily undergo fear conditioning and this has clinical relevance to the genesis of disorders of fear and anxiety (Bouton et al., 2001).

But an even more compelling reason for the success of fear conditioning derives from the observation that brain structures known to be important for memory are involved in encoding and storing fear memories. In the 1960s, it was shown that damage to the amygdala impairs conditioned suppression [(i.e. using the Estes-Skinner procedure) Kellicutt \& Schwartzbaum (1963)] and by the early 1970s Robert and Caroline Blanchard had demonstrated an important role for both the amygdala and hippocampus in conditioned freezing behavior (Blanchard \& Blanchard, 1972; Blanchard et al., 1977). Work in the 1980s by LeDoux, Davis and Kapp further explored and defined the amygdaloid contribution to conditioned fear memories expressed by changes in heart rate, blood pressure, freezing and acoustic startle (Kapp et al., 1979; LeDoux et al., 1984; Hitchcock \& Davis, 1986).

In the early 1990 s, three independent laboratories made a critical observation about the different roles of the hippocampus and amygdala in fear conditioning. Whereas the amygdala was found to be critical for learning about both contextual and discrete (e.g. cues) 
stimuli (Phillips \& LeDoux, 1992), the hippocampus was found to have a selective role in fear to contextual stimuli (i.e. learning about where shocks were delivered; Selden et al., 1991; Kim \& Fanselow, 1992; Phillips \& LeDoux, 1992). Moreover, hippocampal damage was found to produce amnesia for shock contexts that mirrored the temporally graded retrograde amnesia for declarative memories observed in humans with hippocampal damage (Kim \& Fanselow, 1992). These highly influential findings propelled fear conditioning into its current status as one of the premier rodent models for studying declarative memory deficits in humans. As a consequence, the task is often heralded as a tool to index both 'hippocampal-dependent' contextual conditioning and 'hippocampal-independent' cue conditioning in the same animal.

With fame comes scrutiny, however, and subsequent research has yielded new and different insights into the relative contribution of the hippocampus and amygdala to Pavlovian fear conditioning. These data challenge the widely accepted views that: (i) the role of the hippocampus in fear conditioning is modality-specific (i.e. selective for contexts as opposed to cues) and (ii) the amygdala is essential for fear memory. These challenges do not invalidate the utility of fear conditioning as a model for understanding the neurobiology of memory, but they do force a more careful consideration of the data obtained from the model. In addition, these data suggest that a range of conditioning parameters and procedures should be used to interrogate the neural circuit underlying fear memory to dissociate learning from performance impairments, for example.

\section{Is the role of the hippocampus in Pavlovian fear conditioning modality-specific?}

Prior to the investigation of the role of the hippocampus in Pavlovian fear conditioning, a rather substantial literature implicated the hippocampus in spatial, configural and contextual learning rather than Pavlovian conditioning per se (O'Keefe \& Nadel, 1978; Schmajuk, 1984; Sutherland \& Rudy, 1989). In eyeblink conditioning, for example, hippocampal damage has no effect on the acquisition of eyeblink CRs but alters the contextual specificity of conditioning (Penick \& Solomon, 1991). Hence, many early theories of hippocampal function suggested that the hippocampus is not involved in forming associations between CSs and USs per se, but has a role in indexing those associations to the contexts in which they occurred (Hirsh, 1974).

But what about learning Pavlovian associations between places and shocks? Although early studies on this problem suggested that rats with hippocampal damage actually exhibit enhanced contextual conditioning (Winocur et al., 1987), many subsequent studies have found impairments in contextual conditioning with hippocampal lesions (Selden et al., 1991; Phillips \& LeDoux, 1992; Kim et al., 1993; Maren \& Fanselow, 1997; Antoniadis \& McDonald, 2000). More recently, however, several reports have failed to find impairments in contextual conditioning with either permanent or temporary hippocampal lesions (Maren et al., 1997; Frankland et al., 1998; Cho et al., 1999; Richmond et al., 1999). For example, we contrasted the effects of neurotoxic lesions of the dorsal hippocampus on conditioned freezing to contexts and tones (Maren et al., 1997). The lesions were made either 1 week before or at several different time points after fear conditioning. In agreement with previous results, we found that posttraining lesions produced a marked, but temporally graded, deficit in conditioned freezing to the conditioning context; that is, lesions made 1 day after conditioning resulted in massive deficits in context freezing whereas lesions made 100 days after conditioning produced mild deficits in freezing. However, hippocampal lesions made 1 week before fear conditioning had absolutely no effect on the acquisition of context fear, whether shocks were signaled by tones or not. Surprisingly, hippocampal lesions produced reliable deficits in freezing to the auditory CS at all training-lesion intervals. In fact, pretraining lesions of the dorsal hippocampus produced a selective deficit in fear to the auditory CS! Many other reports have also observed failures of hippocampal manipulations to affect the acquisition (Matus-Amat et al., 2004) and expression (McNish et al., 1997; Holt \& Maren, 1999) of context fear, and we have found other instances in which hippocampal manipulations produce more potent effects on discrete, as opposed to contextual, stimuli (Maren \& Holt, 2004).

Based on these and other results, the widely held view that the hippocampus is always required for fear conditioning to contexts, and never cues, must be wrong. What then determines when or whether the hippocampus participates in context or cue conditioning? The answer to this question is not entirely clear. With respect to context conditioning, it appears that animals without a hippocampus can learn about contexts by using the elemental cues that compose the context (Maren, 2001b; Rudy et al., 2004). However, it has been argued that rats do not normally favor this strategy, and typically form configural representations of the collection of cues that define a context (Maren et al., 1997). Memories of context formed this way are thereby sensitive to post-training hippocampal damage. Although rats with hippocampal damage can ultimately learn about contexts in which shock occurs (Maren et al., 1997), their ability to do so is trialdependent (Wiltgen et al., 2006). In other words, hippocampal rats only approach normal levels of conditioning with multiple trials, exhibiting reliable deficits in context conditioning when only a single conditioning trial is administered. Although the influence of US intensity (the magnitude of footshock) has not been explored, it may be the case that exceptionally strong shocks might support learning in rats with hippocampal damage even with a single conditioning trial.

The role for the hippocampus in auditory fear conditioning is similarly complicated. It has long been known that when a slight temporal delay is interposed between the CS and US (a so-called 'trace interval'), the hippocampus is essential for the acquisition and retention of CRs to discrete CSs (Solomon et al., 1986; Moyer et al., 1990; Kim et al., 1995; Quinn et al., 2002; Burman et al., 2006). However, the role of the hippocampus in delay conditioning is debated. An important determinant of auditory fear conditioning deficits may be the nature of the test procedure. In an earlier study, we used relatively short CSs (10 s) during the fear conditioning procedure, but then used a long (8-10 min) CS during the retention test (Maren et al., 1997). In this way the durations of the context and tone tests were equated, although we introduced a mismatch in CS duration between training and testing that may have produced a generalization decrement. Because rats with hippocampal damage were able to sustain reasonably high levels of fear early in the CS, we suggested that their failure to maintain fear across the test was due to a generalization decrement. This possibility is consistent with recent data (Quinn et al., 2002).

Another possibility is that the role for the hippocampus in auditory fear conditioning, like contextual conditioning, is dependent on the strength of conditioning. Hippocampal deficits in auditory fear conditioning appear to be pronounced with either weak shocks (even with multiple training trials) or after limited training (even with strong shocks; Quinn et al., 2008). Based on these and other data, it seems unlikely that the modality of the conditional stimuli (contexts vs. tones) defines the role of the hippocampus in the acquisition of fear conditioning. An alternative is that the strength of the memory to these stimuli influences the sensitivity of context or cue memories to 
hippocampal damage. Weak memories appear to be more susceptible to hippocampal lesions than strong memories, at least when the lesions are made prior to training.

The implications of data like these for the widely held view that the hippocampus is required for context, but not tone, conditioning are obvious, but virtually overlooked. In fact, what these experiments reveal is a very narrow set of parameters within which damage to the hippocampus will produce the archetypal 'context, not tone' deficit. Because this deficit is a function of shock intensity, number of training trials, timing of lesion and perhaps other parameters (e.g. trial spacing, CS intensity and duration, US duration), any neural manipulation that alters how a trial is processed could produce a change in the hippocampal dependence of fear conditioning. Surely, laboratory differences in experimental contexts, CS and US properties and so forth, might also alter the degree to which the hippocampus is engaged in fear conditioning. And once a hippocampal deficit is identified, a variety of procedures is required to isolate the nature of the deficit, which could be caused by a failure to encode, consolidate, or retrieve the context memory, an impairment in the expression of fear to diffuse predictors of shock, a failure to associate context representations with shock, a context discrimination deficit and so forth (Gerlai, 2001). It therefore becomes critical to establish the degree to which specific conditioning procedures generate memories that depend on the hippocampus, and then examine the performance of rats with hippocampal dysfunction across a range of conditioning and test parameters to isolate the nature of the deficit.

\section{Is fear conditioning amygdala-dependent?}

In addition to rethinking the role of the hippocampus in fear conditioning, it is important to consider new data that redefine the role of the amygdaloid nuclei in conditioned fear. There are, of course, many studies revealing the critical role for the central, lateral and, in some cases, the basolateral nucleus in the acquisition and expression of conditioned fear across many different sensory modalities and fear behaviors (Davis, 1992; LeDoux, 2000; Maren \& Quirk, 2004; Fanselow \& Poulos, 2005). The anatomical convergence of CS and US information in the lateral nucleus early in the amygdaloid circuit has led to the view that associative learning is mediated by synaptic plasticity in this region (Maren, 1999a; Blair et al., 2001; Schafe et al., 2001; Goosens \& Maren, 2002). Plasticity within the lateral amygdala is believed to potentiate the ability of the CS to excite neurons in the central nucleus and thereby generate conditioned fear responses.

However, recent data challenge the view that the lateral nucleus is essential for Pavlovian fear conditioning. We have found in a series of experiments that pre-training neurotoxic lesions of the basolateral complex (including the lateral, basolateral and basomedial nuclei) dramatically impair, but do not prevent, the acquisition of conditioned freezing to contexts (Maren, 1998, 1999b; Zimmerman et al., 2007). The acquisition of contextual fear occurred when rats were overtrained, a procedure in which they received 75 context-shock trials in an 80-min session. We have observed mixed results on the acquisition of auditory fear conditioning in rats without a basolateral complex. Our early data suggested that basolateral complex lesions prevent auditory fear conditioning (Maren, 1999b), but more recent experiments have found spared auditory conditioning in some cases (Zimmerman et al., 2007). Importantly, overtrained fear memories are supported by the same associative mechanisms (e.g. CS-US associations) as those that underlie conditioned fear after limited training, and animals with basolateral complex lesions or inactivation are sensitive to US inflation manipulations (indicating that rats with lesions learn in the same manner as intact rats; Rabinak \& Maren, in press).

These data indicate that lateral amygdala synaptic plasticity is not essential for Pavlovian fear conditioning, and that other neural systems can learn in the absence of the lateral amygdala. The obvious alternative is the central nucleus of the amygdala, which is another site for the convergence of CS and US information (Paré et al., 2004). To test this hypothesis, we recently examined whether the central nucleus is essential for fear conditioning in animals with basolateral complex lesions (Zimmerman et al., 2007). The results were unambiguous: lesions or temporary inactivation of the central nucleus completely abolished fear conditioning to both auditory and contextual stimuli after overtraining. These lesions reduced, but did not abolish, post-shock freezing during conditioning. This indicates that rats with central nucleus lesions can freeze. Of course, it remains possible that more extensive training, or spaced training over several sessions, would reveal fear conditioning in animals with central nucleus lesions but until that is demonstrated it would appear that the central nucleus, but not lateral nucleus, is essential for Pavlovian fear conditioning.

In these experiments, we also observed that muscimol inactivation of the central nucleus in intact rats prevented the acquisition of fear (Zimmerman et al., 2007). This reveals that the central nucleus is critical for conditioning even in rats with an intact basolateral complex. The essential role for the central nucleus in fear conditioning is supported by other recent data showing that either inhibition of protein kinases or protein synthesis in the central nucleus impairs the consolidation of fear conditioning (Wilensky et al., 2006). We have also shown that NMDA receptor antagonism in the central nucleus blocks the acquisition of conditioned freezing after limited training (Goosens \& Maren, 2003) and central nucleus neurons exhibit NMDA receptor-dependent long-term potentiation (Fu \& Shinnick-Gallagher, 2005; Samson \& Paré, 2005). Collectively, these data indicate that synaptic plasticity mechanisms in the central nucleus are critical for the acquisition and consolidation of fear memories, and this appears to be true even after limited training in otherwise intact rats.

Nonetheless, the central nucleus is not sufficient for the expression of conditioned fear in animals that have received basolateral complex lesions after overtraining. Post-training basolateral complex lesions completely eliminate the expression of conditioned freezing, and rats with such lesions do not exhibit savings of conditioned fear upon reacquisition (Maren, 1999b, 2001a). Hence, although synaptic transmission and plasticity in the central nucleus is apparently critical for the acquisition of conditioned fear, it cannot sustain fear expression in the absence of the lateral amygdala. This raises the exciting possibility that the lateral amygdala projection to the central nucleus (either directly or indirectly) is the essential locus of plasticity for fear conditioning in intact rats. Indeed, this projection exhibits long-term potentiation in vitro (Fu \& Shinnick-Gallagher, 2005). The strongest projection from the lateral to central nucleus traverses the basolateral nucleus, and basolateral nucleus lesions block the acquisition (Goosens \& Maren, 2001; but see Nader et al., 2001) and expression (Anglada-Figueroa \& Quirk, 2005) of conditioned freezing. Relocating the critical locus of synaptic plasticity to the central nucleus challenges the widely held view that the key plasticity for fear conditioning is on thalamoamygdala or corticoamygdala synapses in the lateral nucleus. Rather than playing an essential role in fear conditioning, associative plasticity at lateral nucleus synapses may augment sensory transmission to the central nucleus and other extraamygdaloid (e.g. cortical and hippocampal) targets and thereby facilitate the induction of long-term potentiation at central nucleus synapses. 
These data have important implications for interpreting the effects of amygdala manipulations on conditional fear. Manipulations that have selective effects on the basolateral complex may spare some capacity for learning, particularly when multiple-trial procedures are used. In contrast, manipulations that influence the central nucleus (either alone or in combination with the basolateral complex) may have more dramatic effects on the acquisition and expression of fear memories. Hence, an appreciation of the differential contribution of the basolateral complex and central nucleus to fear conditioning is essential for interpreting the outcome of amygdala dysfunction.

\section{Performance variables}

Many behavioral measures have been used to index conditioned fear, including pupillary dilation, potentiated acoustic startle, hypertension, tachycardia, freezing and hypoalgesia to name a few. Among these measures, conditioned freezing has become the gold standard for assessing conditioned fear in rodents. The measurement of freezing behavior is noninvasive and can be performed inexpensively. Indeed, visual observation and scoring of freezing behavior (continuously or with a time-sampling procedure) has been a reliable method for assessing fear for many years. More recently, several computerized methods for assessing freezing behavior have been introduced, and these methods eliminate the possibility of observer bias one must be concerned about using human scorers (Anagnostaras et al., 2000). As a consequence, freezing is now typically quantified using either video tracking or with force transducers that record chamber displacement induced by an animal's movement.

Although freezing behavior is a well-established index of conditioned fear, other behaviors may compete with freezing under a variety conditions. For example, exploratory behaviors including rearing and locomotion might compete with freezing when animals are introduced to novel contexts. The geometry of the test environment may influence the expression of conditioned freezing (Bolles \& Collier, 1976). Moreover, increases in locomotor activity that are associated with neural manipulations could reduce the performance of freezing behavior without affecting the acquisition of learned fear responses per se (Maren et al., 1998; Anagnostaras et al., 1999). Clearly, reductions in conditioned freezing alone do not imply a deficit in learning and/or memory. As such, it is essential to determine whether nonassociative factors that influence the performance of fear responses, including freezing, contribute to changes in conditional responding when they are observed (Kamprath \& Wotjak, 2004). Assessments of locomotor activity, for example, provide essential information to aid in the interpretation of neural manipulations that alter freezing behavior; we have used this strategy in our work give our reliance on freezing behavior (Maren et al., 1998; Anagnostaras et al., 1999).

Of course, freezing is just one of many behaviors rodents might exhibit in response to a threat. Bolles provided a compelling account of the defensive repertoire of rats and argued that freezing is one of many species-specific defense responses (SSDRs) that might be engaged by aversive stimuli (Bolles, 1970). The rules governing the selection of SSDRs are not known, but some have argued that the topography of the test situation is an important determinant of freezing behavior. That is, in the absence of an escape route, rats tend to freeze in small boxes (Blanchard et al., 1976a,b; but see Fanselow, 1986). An alternative account is that the level of fear determines the nature of the defensive behavior that is engaged in response to a threat (Fanselow \& Lester, 1988). By this view, moderate levels of fear associated with a distal predator might yield freezing behavior to mitigate detection but particularly high levels of fear associated with contact with the predator might actually reduce conditional freezing as animals engage active defensive behaviors (running, biting, escape). In the laboratory, intense USs or many conditioning trials might therefore reduce the expression of freezing (Fanselow, 1984; Leaton \& Borszcz, 1985). Hence, an absence or reduction of freezing associated with a neural manipulation may not necessarily imply a loss of fear, but rather a shift in the SSDR engaged by the animal. Visual observation of an animal's behavior in the conditioning and test situations is therefore a necessary adjunct to automated methods for quantifying immobility. In addition, measuring multiple fear measures, such as freezing and ultrasonic vocalization, provides convergent indices of fear that do not rely on a single response system (Lee et al., 2001; Choi \& Brown, 2003; Lee \& Kim, 2004).

As in any learning paradigm, therefore, deficits in the expression of freezing behavior might be caused by impairments in either learning or performance. One method for gaining leverage on this problem is to determine whether a particular manipulation has a selective effect on the acquisition or expression of freezing to either contextual or discrete stimuli. For instance, Anagnostaras and colleagues observed that mice lacking muscarinic (M1) receptors exhibited a selective enhancement in freezing to contextual stimuli paired with shock (Anagnostaras et al., 2003). These animals also exhibited more rapid forgetting of the contextual memory, exhibiting substantially less freezing behavior than wild-type controls 30 days after the conditioning experience, and failed to exhibit savings. Conditioned freezing in these animals was similar to wild-type controls to a discrete, auditory conditioned stimulus. Hence, a performance account cannot explain the observed differences in conditioned freezing in the knockout animals, insofar as they are both freezing more than or less than the control animals to various stimuli and at various time points relative to training. In this case, the M1 receptor knockouts appear to have enhanced short-term memory for contextual memories but fail to consolidate these memories into long-term memory.

In many cases, selective impairments in contextual vs. auditory freezing have been observed after a variety of manipulations, and these too have been used to argue explanations based on learning deficits (Kim \& Fanselow, 1992; Abel et al., 1997; Corcoran et al., 2002; Murchison et al., 2004). More problematic, however, are cases in which nonselective deficits in contextual and auditory freezing are observed. For example, Rabenstein and colleagues recently reported that mice lacking $\beta$-adducin, an actin-regulating protein implicated in synaptic plasticity and reorganization, exhibited deficits in conditioned freezing to both contexts and tones (Rabenstein et al., 2005). Locomotor activity, habituation of activity, shock sensitivity and visual acuity were normal. Therefore, the deficits in the expression of conditioned freezing were interpreted as a consequence of deficient learning and memory in the knockout mice. However, it is not clear whether the mice were capable of freezing. The authors might have examined conditioned freezing during the conditioning session or unconditioned freezing to a predator odor. If freezing in either instance was normal and high, it would be less likely that the deficits they observed during retention testing were simply due to performance failures. Additional training would assess whether the deficits in conditioned freezing could be overcome with extended training. A persistent deficit in conditioned freezing that was not overcome with extended training would be consistent with either a memory deficit or performance problem. The analysis of other fear responses in this case might inform whether the absence of freezing in the knockout mice was due to an issue with maintaining immobility, a loss of fear or an impairment in associating neutral stimuli with aversive outcomes. $\beta$-Adducin knockouts also exhibited impaired performance on both 
the hidden- and visible-platform versions of the Morris water maze, which is consistent with a memory account but might also be related to other nonassociative deficits the animals have performing the task

\section{The future of fear}

Despite these cautions and caveats, fear conditioning will continue to be an incredibly useful model for understanding the neurobiological basis for rapidly encoded emotional memories. The relevant neural circuits are becoming clear, the economy and scale of the preparation allows for high-throughput testing, and single-trial (or session) conditioning procedures are ideally suited to the analysis of memory consolidation and reconsolidation. This analysis will be most profitable if it proceeds with an appreciation of, on the one hand, the many factors that influence how and where fear memories are encoded in the brain and, on the other, how they are expressed in behavior. Insofar as fear conditioning continues to be used as a behavioral assay for hippocampus and amygdala function, it is imperative that a broader range of conditioning parameters be explored. In this way, we will not only appreciate that disruption of a specific neural circuit affects a behavior on which it is thought to depend, but will also come to better understand how a particular neural (or molecular) function contributes to the memory process under study. That is, when it is observed that a neural manipulation produces an impairment of context freezing, for example, further interrogation might reveal whether the impairment is due to failures to encode, consolidate or retrieve the context memory, associate the context memory with an aversive outcome, discriminate the conditioning context from other places the animal has been, or engage defensive behavior to diffuse predictors of shock and so forth. Without such a sophisticated behavioral approach, fear conditioning becomes an impoverished assay that reveals only the most rudimentary structure-function relationships concerning the architecture of memory.

\section{Acknowledgements}

This study was supported by the NIH (R01MH065961; R01MH073655).

\section{Abbreviations}

$\mathrm{CR}$, conditional response; CS, conditional stimulus; US, unconditional stimulus.

\section{References}

Abel, T., Nguyen, P.V., Barad, M., Deuel, T.A., Kandel, E.R. \& Bourtchouladze, R. (1997) Genetic demonstration of a role for PKA in the late phase of LTP and in hippocampus-based long-term memory. Cell, 88, 615626.

Anagnostaras, S.G., Maren, S., Sage, J.R., Goodrich, S. \& Fanselow, M.S. (1999) Scopolamine and Pavlovian fear conditioning in rats: dose-effect analysis. Neuropsychopharmacol., 21, 731-744.

Anagnostaras, S.G., Josselyn, S.A., Frankland, P.W. \& Silva, A.J. (2000) Computer-assisted behavioral assessment of pavlovian fear conditioning in mice. Learn. Mem., 7, 58-72.

Anagnostaras, S.G., Murphy, G.G., Hamilton, S.E., Mitchell, S.L., Rahnama, N.P., Nathanson, N.M. \& Silva, A.J. (2003) Selective cognitive dysfunction in acetylcholine M1 muscarinic receptor mutant mice. Nat. Neurosci., 6, 5158

Anglada-Figueroa, D. \& Quirk, G.J. (2005) Lesions of the basal amygdala block expression of conditioned fear but not extinction. J. Neurosci., 25, 9680-9685.

Antoniadis, E.A. \& McDonald, R.J. (2000) Amygdala, hippocampus and discriminative fear conditioning to context. Behav. Brain Res., 108, 1-19.
Blair, H.T., Schafe, G.E., Bauer, E.P., Rodrigues, S.M. \& LeDoux, J.E. (2001) Synaptic plasticity in the lateral amygdala: A cellular hypothesis of fear conditioning. Learn. Mem., 8, 229-242.

Blanchard, D.C. \& Blanchard, R.J. (1972) Innate and conditioned reactions to threat in rats with amygdaloid lesions. J. Comp. Physiol. Psychol., 81, 281290

Blanchard, R.J., Fukunaga, K.K. \& Blanchard, D.C. (1976a) Environmentalcontrol of defensive reactions to a cat. Bull. Psychon. Soc., 8, 179-181.

Blanchard, R.J., Fukunaga, K.K. \& Blanchard, D.C. (1976b) Environmentalcontrol of defensive reactions to footshock. Bull. Psychon. Soc., 8, 129130

Blanchard, D.C., Blanchard, R.J., Lee, E.M.C. \& Fukunaga, K.K. (1977) Movement arrest and the hippocampus. Physiol. Psychol., 5, 331-335.

Bolles, R.C. (1970) Species-specific defense reactions and avoidance learning. Psychol. Rev., 77, 32-48.

Bolles, R.C. \& Collier, A.C. (1976) Effect of predictive cues on freezing in rats. Anim. Learn. Behav., 4, 6-8.

Bouton, M.E., Mineka, S. \& Barlow, D.H. (2001) A modern learning theory perspective on the etiology of panic disorder. Psychol. Rev., 108, 4-32.

Burman, M.A., Starr, M.J. \& Gewirtz, J.C. (2006) Dissociable effects of hippocampus lesions on expression of fear and trace fear conditioning memories in rats. Hippocampus, 16, 103-113.

Cho, Y.H., Friedman, E. \& Silva, A.J. (1999) Ibotenate lesions of the hippocampus impair spatial learning but not contextual fear conditioning in mice. Behav. Brain Res., 98, 77-87.

Choi, J.S. \& Brown, T.H. (2003) Central amygdala lesions block ultrasonic vocalization and freezing as conditional but not unconditional responses. J. Neurosci., 23, 8713-8721.

Corcoran, K.A., Lu, Y., Turner, R.S. \& Maren, S. (2002) Overexpression of hAPPswe impairs rewarded alternation and contextual fear conditioning in a transgenic mouse model of Alzheimer's disease. Learn. Mem., 9, 243-252.

Davis, M. (1992) The role of the amygdala in fear and anxiety. Annu. Rev Neurosci., 15, 353-375.

Estes, W.K. \& Skinner, B.F. (1941) Some quantitative properties of anxiety. J. Exp. Psychol., 29, 390

Fanselow, M.S. (1984) Shock-induced analgesia on the formalin test: effects of shock severity, naloxone, hypophysectomy, and associative variables. Behav. Neurosci., 98, 79-95.

Fanselow, M.S. (1986) Associative vs topographical accounts of the immediate shock-freezing deficit in rats: Implications for the response selection rules governing species-specific defensive reactions. Learn. Motiv, 17, 16-39.

Fanselow, M.S. \& Lester, L.S. (1988) A functional behavioristic approach to aversively motivated behavior: Predatory imminence as a determinant of the topography of defensive behavior. In Bolles, R.C. \& Beecher, M.D. (eds), Evolution and Learning. Erlbaum, Hillsdale, NJ, pp. 185-211.

Fanselow, M.S. \& Poulos, A.M. (2005) The neuroscience of mammalian associative learning. Annu. Rev. Psychol., 56, 207-234.

Frankland, P.W., Cestari, V., Filipkowski, R.K., McDonald, R.J. \& Silva, A.J. (1998) The dorsal hippocampus is essential for context discrimination but not for contextual conditioning. Behav. Neurosci., 112, 863-874.

Fu, Y. \& Shinnick-Gallagher, P. (2005) Two intra-amygdaloid pathways to the central amygdala exhibit different mechanisms of long-term potentiation. J. Neurophysiol., 93, 3012-3015.

Gerlai, R. (2001) Behavioral tests of hippocampal function: simple paradigms complex problems. Behav. Brain Res., 125, 269-277.

Goosens, K.A. \& Maren, S. (2001) Contextual and auditory fear conditioning are mediated by the lateral, basal, and central amygdaloid nuclei in rats. Learn. Mem., 8, 148-155.

Goosens, K.A. \& Maren, S. (2002) Long-term potentiation as a substrate for memory: evidence from studies of amygdaloid plasticity and Pavlovian fear conditioning. Hippocampus, 12, 592-599.

Goosens, K.A. \& Maren, S. (2003) Pretraining NMDA receptor blockade in the basolateral complex, but not the central nucleus, of the amygdala prevents savings of conditional fear. Behav. Neurosci., 117, 738-750.

Hirsh, R. (1974) The hippocampus and contextual retrieval of information from memory: a theory. Behav. Biol., 12, 421-444.

Hitchcock, J. \& Davis, M. (1986) Lesions of the amygdala, but not of the cerebellum or red nucleus, block conditioned fear as measured with the potentiated startle paradigm. Behav. Neurosci., 100, 11-22.

Holt, W. \& Maren, S. (1999) Muscimol inactivation of the dorsal hippocampus impairs contextual retrieval of fear memory. J. Neurosci., 19, 9054-9062.

Kamprath, K. \& Wotjak, C.T. (2004) Nonassociative learning processes determine expression and extinction of conditioned fear in mice. Learn. Mem., 11, 770-786. 
Kapp, B.S., Frysinger, R.C., Gallagher, M. \& Haselton, J.R. (1979) Amygdala central nucleus lesions: effect on heart rate conditioning in the rabbit Physiol. Behav., 23, 1109-1117.

Kellicutt, M.H. \& Schwartzbaum, J.S. (1963) Formation of a conditioned emotional response (CER) following lesions of the amygdaloid complex in rats. Psychol. Rep., 12, 351-358.

Kim, J.J. \& Fanselow, M.S. (1992) Modality-specific retrograde amnesia of fear. Science, 256, 675-677.

Kim, J.J., Rison, R.A. \& Fanselow, M.S. (1993) Effects of amygdala hippocampus, and periaqueductal gray lesions on short- and long-term contextual fear. Behav. Neurosci., 107, 1093-1098.

Kim, J.J., Clark, R.E. \& Thompson, R.F. (1995) Hippocampectomy impairs the memory of recently, but not remotely, acquired trace eyeblink conditionedresponses. Behav. Neurosci., 109, 195-203.

Leaton, R.N. \& Borszcz, G.S. (1985) Potentiated startle - its relation to freezing and shock-intensity in rats. J. Exp. Psychol. Anim. B, 11, 421-428

LeDoux, J.E. (2000) Emotion circuits in the brain. Annu. Rev. Neurosci., 23, $155-184$.

LeDoux, J.E., Sakaguchi, A. \& Reis, D.J. (1984) Subcortical efferent projections of the medial geniculate nucleus mediate emotional responses conditioned to acoustic stimuli. J. Neurosci., 4, 683-698.

Lee, T. \& Kim, J.J. (2004) Differential effects of cerebellar, amygdalar, and hippocampal lesions on classical eyeblink conditioning in rats. J. Neurosci., 24, 3242-3250

Lee, H.J., Choi, J.S., Brown, T.H. \& Kim, J.J. (2001) Amygdalar NMDA receptors are critical for the expression of multiple conditioned fear responses. J. Neurosci., 21, 4116-4124.

Maren, S. (1998) Overtraining does not mitigate contextual fear conditioning deficits produced by neurotoxic lesions of the basolateral amygdala. J. Neurosci, 18, 3088-3097.

Maren, S. (1999a) Long-term potentiation in the amygdala: a mechanism for emotional learning and memory. Trends Neurosci., 22, 561-567.

Maren, S. (1999b) Neurotoxic basolateral amygdala lesions impair learning and memory but not the performance of conditional fear in rats. J. Neurosci., 19 , 8696-8703.

Maren, S. (2001a) Is there savings for Pavlovian fear conditioning after neurotoxic basolateral amygdala lesions in rats? Neurobiol. Learn. Mem., 76 , 268-283.

Maren, S. (2001b) Neurobiology of Pavlovian fear conditioning. Annu. Rev. Neurosci., 24, 897-931.

Maren, S. \& Fanselow, M.S. (1997) Electrolytic lesions of the fimbria/fornix, dorsal hippocampus, or entorhinal cortex produce anterograde deficits in contextual fear conditioning in rats. Neurobiol. Learn. Mem., 67, 142-149.

Maren, S. \& Holt, W.G. (2004) Hippocampus and Pavlovian fear conditioning in rats: muscimol infusions into the ventral, but not dorsal, hippocampus impair the acquisition of conditional freezing to an auditory conditional stimulus. Behav. Neurosci., 118, 97-110.

Maren, S. \& Quirk, G.J. (2004) Neuronal signalling of fear memory. Nat. Rev. Neurosci., 5, 844-852.

Maren, S., Aharonov, G. \& Fanselow, M.S. (1997) Neurotoxic lesions of the dorsal hippocampus and Pavlovian fear conditioning in rats. Behav. Brain Res., 88, 261-274.

Maren, S., Anagnostaras, S.G. \& Fanselow, M.S. (1998) The startled seahorse: Is the hippocampus necessary for contextual fear conditioning? Trends Cogn Sci., 2, 39-42.

Matus-Amat, P., Higgins, E.A., Barrientos, R.M. \& Rudy, J.W. (2004) The role of the dorsal hippocampus in the acquisition and retrieval of context memory representations. J. Neurosci., 24, 2431-2439.

McNish, K.A., Gewirtz, J.C. \& Davis, M. (1997) Evidence of contextual fear after lesions of the hippocampus: a disruption of freezing but not fearpotentiated startle. J. Neurosci., 17, 9353-9360.

Moyer, J.R. Jr, Deyo, R.A. \& Disterhoft, J.F. (1990) Hippocampectomy disrupts trace eye-blink conditioning in rabbits. Behav. Neurosci., 104, $243-$ 252
Murchison, C.F., Zhang, X.Y., Zhang, W.P., Ming, O.Y., Lee, A. \& Thomas, S.A. (2004) A distinct role for norepinephrine in memory retrieval. Cell, 117, 131-143.

Nader, K., Majidishad, P., Amorapanth, P. \& LeDoux, J.E. (2001) Damage to the lateral and central, but not other, amygdaloid nuclei prevents the acquisition of auditory fear conditioning. Learn. Mem., 8, 156-163.

O'Keefe, J. \& Nadel, L. (1978) The hippocampus as a cognitive map. Oxford University Press, Clarendon Press, Oxford.

Paré, D., Quirk, G.J. \& Ledoux, J.E. (2004) New vistas on amygdala networks in conditioned fear. J. Neurophysiol., 92, 1-9.

Penick, S. \& Solomon, P.R. (1991) Hippocampus, context, and conditioning. Behav. Neurosci., 105, 611-617.

Phillips, R.G. \& LeDoux, J.E. (1992) Differential contribution of amygdala and hippocampus to cued and contextual fear conditioning. Behav. Neurosci., 106, 274-285.

Quinn, J.J., Oommen, S.S., Morrison, G.E. \& Fanselow, M.S. (2002) Posttraining excitotoxic lesions of the dorsal hippocampus attenuate forward trace, backward trace, and delay fear conditioning in a temporally specific manner. Hippocampus, 12, 495-504.

Quinn, J.J., Wied, H.M., Ma, Q.D., Tinsley, M.R. \& Fanselow, M.S. (2008) Dorsal hippocampus involvement in delay fear conditioning depends upon the strength of the tone-footshock association. Hippocampus, 18, 640-654.

Rabenstein, R.L., Addy, N.A., Caldarone, B.J., Asaka, Y., Gruenbaum, L.M., Peters, L.L., Gilligan, D.M., Fitzsimonds, R.M. \& Picciotto, M.R. (2005) Impaired synaptic plasticity and learning in mice lacking beta-adducin, an actin-regulating protein. J. Neurosci., 25, 2138-2145.

Rabinak, C.A. \& Maren, S. (in press) Associative structure of fear memory after basolateral amygdala lesions in rats. Behav. Neurosci.

Richmond, M.A., Yee, B.K., Pouzet, B., Veenman, L., Rawlins, J.N., Feldon, J. \& Bannerman, D.M. (1999) Dissociating context and space within the hippocampus: effects of complete, dorsal, and ventral excitotoxic hippocampal lesions on conditioned freezing and spatial learning. Behav. Neurosci., 113, 1189-1203.

Rudy, J.W., Huff, N.C. \& Matus-Amat, P. (2004) Understanding contextual fear conditioning: insights from a two-process model. Neurosci. Biobehav. Rev., 28, 675-685.

Samson, R.D. \& Paré, D. (2005) Activity-dependent synaptic plasticity in the central nucleus of the amygdala. J. Neurosci., 25, 1847-1855.

Schafe, G.E., Nader, K., Blair, H.T. \& LeDoux, J.E. (2001) Memory consolidation of Pavlovian fear conditioning: a cellular and molecular perspective. Trends Neurosci., 24, 540-546.

Schmajuk, N.A. (1984) Psychological theories of hippocampal function. Physiol. Psychol., 12, 166-183.

Selden, N.R., Everitt, B.J., Jarrard, L.E. \& Robbins, T.W. (1991) Complementary roles for the amygdala and hippocampus in aversive conditioning to explicit and contextual cues. Neuroscience, 42, 335-350.

Solomon, P.R., Vander Schaaf, E.R., Thompson, R.F. \& Weisz, D.J. (1986) Hippocampus and trace conditioning of the rabbit's classically conditioned nictitating membrane response. Behav. Neurosci., 100, 729-744.

Sutherland, R.J. \& Rudy, J.W. (1989) Configural association theory: The role of the hippocampal formation in learning, memory, and amnesia. Psychobiol., 17, 129-144

Wilensky, A.E., Schafe, G.E., Kristensen, M.P. \& LeDoux, J.E. (2006) Rethinking the fear circuit: the central nucleus of the amygdala is required for the acquisition, consolidation, and expression of Pavlovian fear conditioning. J. Neurosci., 26, 12387-12396.

Wiltgen, B.J., Sanders, M.J., Anagnostaras, S.G., Sage, J.R. \& Fanselow, M.S. (2006) Context fear learning in the absence of the hippocampus. J. Neurosci. 26, 5484-5491

Winocur, G., Rawlins, J.N. \& Gray, J.A. (1987) The hippocampus and conditioning to contextual cues. Behav. Neurosci., 101, 617-625.

Zimmerman, J.M., Rabinak, C.A., McLachlan, I.G. \& Maren, S. (2007) The central nucleus of the amygdala is essential for acquiring and expressing conditional fear after overtraining. Learn. Mem., 14, 634-644. 